\title{
Volumetric Properties of (Difurylmethane + an Amide) Binary Mixtures at 293.15K, 298.15K and 303.15 K
}

\author{
Belcher Fulele, Wilfred A. A. Ddamba \\ Department of Chemistry, Faculty of Science, University of Botswana, Gaborone, Botswana
}

Email address:

fuleleb@mopipi.ub.bw (B. Fulele), ddambawa@mopipi.ub.bw (W. A. A. Ddamba)

To cite this article:

Belcher Fulele, Wilfred A. A. Ddamba. Volumetric Properties of (Difurylmethane + an Amide) Binary Mixtures at 293.15K, 298.15K and 303.15 K. American Journal of Physical Chemistry. Vol. 6, No. 2, 2017, pp. 6-19. doi: 10.11648/j.ajpc.20170602.11

Received: February 7, 2017; Accepted: March 1, 2017; Published: March 21, 2017

\begin{abstract}
Densities $(\rho)$ of pure difuryl methane (DFM), N-methylformamide (NMF), N-ethylformamide (NEF), N,Ndimethylformamide (DMF) and N,N-dimethylacetamide (DMA) and those of [DFM + (NMF or NEF or DMF or DMA] binary mixtures over the entire composition range, have been measured at $(T=293.15,298.15$ and 303.15$) \mathrm{K}$ and atmospheric pressure. Excess molar volumes $\left(V_{m}^{E}\right)$, of each binary system were calculated and correlated by the Redlich-Kister equation. $V_{m}^{E}$ values for each binary system were negative at each of the three temperatures investigated $(T=293.15,298.15$ and 303.15$)$ $\mathrm{K}$. The $V_{m}^{E}$ values decreased with temperature increase for each of the binary systems investigated. Sign and magnitude of $V_{m}^{E}$ are discussed in-terms of type and nature of intermolecular interactions that occur in a binary mixture. The $V_{m}^{E}$ data have been used to derive excess partial molar volumes $\left(V_{i}^{E}\right)$, limiting excess partial molar volumes $\left(V_{i}^{E, \infty}\right)$ and limiting partial molar volumes at infinite dilute region $\left(V_{i}^{\infty}\right)$ of each component of the binary mixture. Results are discussed in terms of possible intermolecular interactions and structural effects that occur in the binary mixtures.
\end{abstract}

Keywords: Difurylmethane, an Amide, Excess Molar Volume, Excess Partial Molar Volume, Binary Mixtures,

Dipole-Dipole Interactions, Hydrogen Bond

\section{Introduction}

In previous papers we have reported studies on volumetric $[1,2,3]$ and acoustic properties [4] of binary mixtures containing difuryl methane (DFM) and $\left(\mathrm{C}_{1}-\mathrm{C}_{6}\right) n$-alkanols at various temperatures. These investigations provided a deep insight with respect to understanding of the DFM / $\left(C_{1}-C_{6}\right) n$ alkanols intermolecular interactions and revealed significant deviation from ideal behavior which was attributed to chemical and structural interaction effects. The present investigations are focused on the study of intermolecular interactions in binary systems containing DFM with $\mathrm{N}$ methylformamide (NMF), N-ethylformamide (NEF), N,Ndimethylformamide (DMF) and N,N -dimethylacetamide (DMA) over the entire composition range at $(\mathrm{T}=293.15$, 298.15 and 303.15) $\mathrm{K}$ and atmospheric pressure. DFM is an aprotic dipolar substance whose liquid structure is determined by the dipole-dipole interactions [1-3] and has the potential for use in solvent extraction processes for separating polar organic substances from aqueous media. On the other hand secondary amides (NMF and NEF) are strongly self-associated through hydrogen bonding and dipole-dipole interactions (NMF, $\mu=3.86 \mathrm{D}$; NEF, $\mu=3.80 \mathrm{D}$ at $298.15 \mathrm{~K})$ [5-9]. This association is reported to decrease with the size of alkyl group in the amide molecule $[6,10]$. DMF and DMA lack any significant liquid structural effects due to the absence of hydrogen bonding. However, DMF and DMA possess strong electron pair donating and accepting abilities due to the presence of large dipoles in their structure (DMF, $\mu=3.86$; DMA, $\mu=3.72 \mathrm{D}$ at 298.15K) [6]. Thus significant intermolecular interactions between unlike components in binary mixtures of DFM with NMF or NEF or DMF or DMA would be expected to occur. A survey of the literature has revealed no study on the volumetric behavior of these binary systems. Therefore the present investigation was expected to reveal data on the intermolecular interactions in (DFM + an amide) binary systems.

In the present study we report densities $\rho$, of (DFM + NMF or NEF or DMF or DMA) binary mixtures at ( $\mathrm{T}=$ 293.15, 298.15 and 303.15)K and atmospheric pressure over the entire composition range. From the experimental density 
measurements, the excess molar volumes $V_{m}^{E}$ were calculated at each temperature and composition. For each of the binary mixture investigated, the Redlich-Kister polynomial equation of an appropriate degree was fitted to the $V_{m}^{E}$ data. From the $V_{m}^{E}$ data, derived properties which included excess partial molar volume, $\bar{V}_{i}^{E}$, limiting partial molar volume, $V_{i}^{\infty}$ and limiting excess partial molar volume $\bar{V}_{i}^{E, \infty}$ were calculated. The magnitude and sign of $V_{m}^{E}$ as well as derived properties $\left(V_{m}^{E}, \bar{V}_{i}^{E}, \bar{V}_{i}^{E, \infty}\right.$ ) provided a better understanding of the intermolecular interactions existing between the various species in each of (DFM + NMF or NEF or DMF or DMA) binary mixtures.

\section{Experimental}

\subsection{Materials}

The following chemicals were used: $n$-hexane (Merck Co., $\geq 98.0 \%$ ), NMF, (Sigma-Aldrich chemicals, 99.0\%), NEF (Fluka, analytical reagent 99.0\%), DMA, (Sigma-Aldrich chemicals, 99.0\%), DMF, (BDH chemicals, analytical reagent, 99.5\%). All amides used in the present study (NMF, NEF, DMA and DMF) were analytical reagents of good quality as reflected by the measured density in comparison to the corresponding $\rho$-literature data (Table 1). Before use, each of the amides was stored over $0.40 \mathrm{~nm}$ molecular sieves for $74 h$ to remove water content, as much as possible. DFM was prepared as described elsewhere [20] and its purity was confirmed by ${ }^{1} \mathrm{H}-\mathrm{NMR}$, density measurements and elemental analysis. All purified organic liquids were stored in brown glass bottles. The conductivity of ultrapure water used to calibrate the densimeter was always less than $1.0 \times 10^{-6} \mathrm{~S}_{-\mathrm{cm}^{-1}}$.

\subsection{Apparatus and Procedure}

Each one of the (DFM + NMF or NEF or DMF or DMA) binary mixtures was prepared by weighing appropriate amounts of purified DFM and the corresponding co-solvent (amide) on an analytical balance $(\Delta m= \pm 0.0001 \mathrm{~g})$, by syringing each component into a teflon stoppered flask. Pure components were first separately degassed in an ultrasonic bath shortly before sample preparations. All (DFM + NMF or NEF or DMF or DMA) binary mixtures were completely miscible over the entire composition range. The average uncertainty in solution composition expressed in mole fraction was found to be less than $8.5 \times 10^{-4}$. Density measurements were performed at atmospheric pressure with an Anton Paar DMA 4500 vibrating-tube densimeter at the experimental temperature. The densimeter was first calibrated with pure water and benzene as reference liquids. A sample volume of not more than $1.0 \mathrm{~cm}^{3}$ was needed to fill the densimeter cell and thermal equilibrium was attained quickly. The temperature of the sample was controlled electrically by means of a built-in thermostat (a semiconductor Peltier element and a resistance thermometer temperature control system) and was measured with an accuracy of $\pm 0.01 \mathrm{~K}$. The densimeter was calibrated after each set of four sample measurements to offset possible instrument drift. A linear relation between the density of the fluid and the square of the vibrating period $\tau$, $\left(\rho=A+B \tau^{2}\right)$, was assumed. Buoyancy corrections were made by taking into account the air density at each of the three temperatures, the barometric pressure, and the relative humidity. Under such conditions triplet density measurements of each sample were reproducible to within $\pm 0.01 \mathrm{~kg}-\mathrm{m}^{-3}$. The reliability of experimental measurements of density was ascertained by comparing the experimental data of pure liquids with the corresponding literature values at the studied temperatures.

\section{Results and Discussion}

\subsection{Excess Molar Volumes}

Excess molar volumes, $V_{m}^{E}$, were calculated for each of (DFM + NMF or NEF or DMF or DMA) binary systems from density measurements using equation (1):

$$
V_{m}^{E}=\mathrm{V}_{\mathrm{m}}-V_{m}^{i d}=\mathrm{M} / \rho-\sum \mathrm{x}_{\mathrm{i}} \mathrm{M}_{\mathrm{i}} / \rho_{\mathrm{i}}
$$

where $M$ is the molar mass of the mixture, which is the mole fraction weighted adduct of the molar masses of the two pure components in each binary mixture, i.e. $\left(M=M_{1} x_{1}+M_{2} x_{2}\right)$. $V_{m}^{i d}$ is the ideal molar volume, $\rho$ is the density of the mixture, and $x_{i}, M_{i}$ and $\rho_{i}$ are respectively the mole fraction, the molar mass and the density of the pure liquid component $i$. The experimental $V_{m}^{E}$ values of each of the (DFM + NMF or NEF or DMF or DMA) binary systems (Table 2) were leastsquares fitted to the Redlich-Kister polynomial equation [21]:

$$
V_{m}^{E}=x_{1} x_{2} \sum_{k=0}^{k=n} A_{k}\left(1-2 x_{2}\right)^{k}
$$

where $n$ is the order of the polynomial, $x_{l}$ is the mole fraction of the amide and $x_{2}$ is the mole fraction of DFM. For each binary system at a specified temperature, the degree of the polynomial and the number of $A_{k}$ coefficients in Equation (2) were fixed by testing the statistical significance of including each further term using an F-test at a $99.5 \%$ confidence level. The optimized number of regression coefficients $A_{k}$ for each of the four binary systems are listed in Table 4 along with the corresponding standard deviation $\sigma\left(V_{m}^{E}\right)$, defined by Equation (3):

$$
\sigma\left(V_{m}^{E}\right)=\left[\sum\left(V_{m, \exp }^{E}-V_{m, c a l c}^{E}\right)^{2} /(\mathrm{N}-\mathrm{n})\right]^{1 / 2}
$$

where $N$ is the number of data points and $n$ represents number of regression coefficients.

Excess thermodynamic functions such as the excess molar volume $V_{m}^{E}$, measure the extent of deviation from the ideal solution behavior. In addition, the temperature dependent measurements of $V_{m}^{E}$ in general, are of interest for a better understanding of the structural effects between unlike molecules in a binary mixture. Figure 1 represent the experimental $V_{m}^{E}$ vs $x_{2}$ isotherms for (DFM + NMF or NEF or DMF or DMA) binary systems at $298.15 \mathrm{~K}$. Negative $V_{m}^{E}$ values were observed over the entire composition range at the three temperatures examined for each binary system. The $V_{m}^{E}$ vs $x_{2}$ isotherms for all [DFM + (NMF or NEF or DMF or DMA)] binary systems show $V_{m(\min )}^{E}$ values at $x_{2} \approx 0.5$, which 
in each case corresponds to formation of a 1:1, DFM: amide associates. The sequence in the negative magnitude of $V_{m}^{E}$ (min) values at $298.15 \mathrm{~K}$ is: $\mathrm{DMA}>\mathrm{DMF}>\mathrm{NEF}>\mathrm{NMF}$ and were $-0.739 \mathrm{~cm}^{3} \mathrm{~mol}^{-1}$ (at $\left.x_{2}=0.501\right),-0.697 \mathrm{~cm}^{3} \mathrm{~mol}^{-1}$ (at $x_{2}=$ 0.501 ), $-0.336 \mathrm{~cm}^{3} \mathrm{~mol}^{-1}$ (at $x_{2}=0.519$ ), $-0.249 \mathrm{~cm}^{3} \mathrm{~mol}^{-1}$ (at $\left.x_{2}=0.449\right)$, respectively. Negative values for $V_{m}^{E}$ vs $x_{2}$ isotherms suggest the presence of specific intermolecular interactions between DFM and amide molecules in each binary system.

Assuming the DFM-amide, dipole-dipole intermolecular interactions as the dominant effects, a possible qualitative explanation for the observed volumetric behavior is as follows starting with (DFM + NMF or NEF) binary systems. The liquid structure of the pure DFM is determined by the dipole-dipole interactions [2]. On the other hand, molecules of secondary amides are self-associated through hydrogen bonding due to the presence of a strong proton-acceptor group $(\mathrm{C}=\mathrm{O})$ and a proton-donor group $(-\mathrm{NH})$ in their molecules and the dipole-dipole intermolecular interactions $[6,8,9]$. Thus the mixing of DFM with the secondary amides to form [DFM + (NMF or NEF)] binary systems would result in mutual disruption of the dipole-dipole associates in the DFM liquid structure and the hydrogen-bonded aggregates as well as the dipole-dipole interactions in pure NMF or NEF, which would contribute positively to $V_{m}^{E}$ values. On the other hand, the intermolecular associative dipole-dipole interactions between the amide molecules (NMF or NEF) and the polarized $\pi$-electron system of the furan rings in DFM would lead to closer molecular packing which would contribute to a decrease in $V_{m}^{E}$ values. From the negative

Table 1. Densities, $\rho\left(\mathrm{kg}^{-\mathrm{m}^{-3}}\right)$ of pure components at $(T=293.15,298.15$ and 303.15) $\mathrm{K}$.

\begin{tabular}{|c|c|c|c|}
\hline Compound & $\mathbf{T} / \mathbf{K}$ & $\rho\left(\mathrm{kg} / \mathrm{m}^{3}\right)$ & \\
\hline & & $\operatorname{Exp}$ & Lit \\
\hline \multirow[t]{5}{*}{$N$-methylformamide } & 293.15 & 1003.84 & $1003.18^{\mathrm{a}}$ \\
\hline & 298.15 & 999.5 & $999.29^{\mathrm{b}}$ \\
\hline & & & $999.20^{c}$ \\
\hline & 303.15 & 995.15 & $994.80^{d}$ \\
\hline & & & $994.65^{e}$ \\
\hline \multirow[t]{3}{*}{$N$-Ethylformamide } & 293.15 & 951.87 & $951.59^{f}$ \\
\hline & 298.15 & 947.78 & $947.48^{f}$ \\
\hline & 303.15 & 943.68 & $943.29^{f}$ \\
\hline \multirow[t]{7}{*}{$N, N$ Dimethylformamide } & 293.15 & 949.04 & $948.51^{c}$ \\
\hline & & & $\begin{array}{l}948.73^{g} \\
944.09^{h}\end{array}$ \\
\hline & 298.15 & 944.3 & $\begin{array}{l}944.09^{h} \\
944.40^{b}\end{array}$ \\
\hline & & & $944.60^{i, e}$ \\
\hline & & & $943.85^{j}$ \\
\hline & 303.15 & 939.53 & $938.88^{k}$ \\
\hline & & & $939.83^{l}$ \\
\hline \multirow[t]{7}{*}{$N, N$-Dimethylacetamide } & 293.15 & 941.53 & $941.02^{a}$ \\
\hline & & & $941.50^{g}$ \\
\hline & 298.15 & 936.94 & $936.41^{a}$ \\
\hline & & & $936.50^{i, l}$ \\
\hline & & & $936.34^{g}$ \\
\hline & 303.15 & 932.33 & $931.79^{b}$ \\
\hline & & & $931.69^{g}$ \\
\hline \multirow[t]{3}{*}{ Difurylmethane } & 293.15 & 1095.68 & $1095.94^{m}$ \\
\hline & 298.15 & 1090.63 & $1090.88^{m}$ \\
\hline & 303.15 & 1085.57 & $1085.82^{m}$ \\
\hline
\end{tabular}

${ }^{a}$ Ref.[5], ${ }^{b}$ Ref.[7], ${ }^{c}$ Ref. [15], ${ }^{d}$ Ref. [12], ${ }^{e}$ Ref. [16],${ }^{f}$ Ref.[8], ${ }^{g}$ Ref. [14], ${ }^{h}$ Ref. [17], ${ }^{i}$ Ref. [11], ${ }^{j}$ Ref.[19], ${ }^{k}$ Ref.[13], ${ }^{\prime}$ Ref. [18], ${ }^{m}$ Ref. [1].
$V_{m}^{E}$ experimental values observed, formation of DFMNMF or DFM-NEF associates supersedes the positive contribution to $V_{m}^{E}$, arising from the rupture of the liquid structures in the pure unlike components of (DFM + NMF or NEF) binary systems. The negative $V_{m}^{E}$ values thus indicate that DFM and NMF/NEF molecules are more efficiently packed in the binary mixtures than in their pure states. Spectroscopic and dielectric studies confirm the strong self-association of secondary amides through $\mathrm{N}$ $\mathrm{H} \cdots \mathrm{O}=\mathrm{C}$ hydrogen bonds which has been reported to persist even in the infinite dilute amide solutions [8, 9]. It is possible that addition of DFM to NMF or NEF may only partially disrupt the hydrogen-bonded aggregates in each of the secondary amides. The hydrogen bond density in secondary amides would be expected to decrease with increase in the alkyl chain length and the degree of hydrogen bonding in pure NMF would be greater than in NEF. As a consequence of this dilution effect on the hydrogen bond density arising from a bigger $-\mathrm{CH}_{2} \mathrm{CH}_{3}$ group in NEF, the disruption of the hydrogen-bonds in NEF when (DFM + NEF) binary mixture is formed, a bigger proportion of NEF molecules would be released for formation of DFM: NEF, 1:1 associates. This would result in a more efficient intermolecular interaction as reflected in the more negative $V_{m}^{E}$ values for $(\mathrm{DFM}+\mathrm{NEF})$ compared to (DFM + NMF) binary system.

In pure DMF or DMA, molecules are weakly associated via non-specific van der Waal interactions and dipole-dipole interactions [6]. When each of the (DFM + DMF or DMA) binary mixture is formed, negative $V_{m}^{E} v s \quad x_{2}$ isotherms observed are attributed to strong DFM: DMF and DFM: DMA, dipole-dipole intermolecular interactions. It is also observed that the $V_{m}^{E}$ values are more negative for (DFM + DMA) than in (DFM + DMF) binary system over the entire $x_{2}$ - range. The lone-pair on the nitrogen atom in both DMF and DMA is protected by the two $-\mathrm{CH}_{3}$ groups rendering it less accessible. However the presence of the $-\mathrm{CH}_{3}$ group at the carbon atom of the $\mathrm{C}=\mathrm{O}$ group has the effect of increasing the electron density at the oxygen atom in DMA [11] compared to the hydrogen at the same position in DMF. This effect is reflected in the greater proton affinity at the oxygen site of DMA (904.04 $\mathrm{kJ} \mathrm{mol}^{-1}$ ) compared to that in DMF (879.02 $\left.\mathrm{kJ} \mathrm{mol}^{-1}\right)$. [22]. Consequently, this effect may contribute to a more favorable DFM: DMA, dipole - dipole interaction, which results in more negative $V_{m}^{E}$ values for (DFM + DMA) compared to (DFM + DMF) binary system.

Another possible contribution to negative $V_{m}^{E}$ values may arise from structural effects due to the geometrical interstitial accommodation of the smaller amide component molecules $(\mathrm{NMF}=59.09, \mathrm{NEF}=77.12, \mathrm{DMF}=77.41$ and DMA $=92.98 \mathrm{~cm}^{3} \mathrm{~mol}^{-1}$ ) into cavities created by molecules of the larger DFM component $\left(\mathrm{DFM}=135.85 \mathrm{~cm}^{3} \mathrm{~mol}^{-1}\right.$ at $298.15 \mathrm{~K})$ when (DFM + NMF or NEF or DMF or DMA) binary systems are formed. This effect to a more closely packed liquid structure in the corresponding binary mixture. Geometrical effects also may result from differences in shape and molar free volumes of unlike pure components [6]. 
Table 2. Experimental densities $\rho\left(\mathrm{kg} \mathrm{m}^{-3}\right)$, excess molar volume, $V_{m}^{E}$ and excess partial molar volumes $\bar{V}_{i}^{E}\left(\mathrm{~cm}^{3}\right.$ mol $\left.{ }^{-1}\right)$ for $(D F M+N M F$ or $N E F$ or DMF or $D M A)$ binary mixtures at $(T=293.15,298.15$ and 303.15) $\mathrm{K}$.

\begin{tabular}{|c|c|c|c|c|}
\hline $\mathbf{x}_{2}$ & $\rho$ & $V_{m}^{E}$ & $\mathbf{V}_{1}{ }^{\mathrm{E}}$ & $\mathbf{V}_{2}{ }^{\mathrm{E}}$ \\
\hline & & $\begin{array}{l}(\mathrm{DFM}+\mathrm{NMF}) \\
\mathrm{T}=293.15 \mathrm{~K}\end{array}$ & & \\
\hline 0.00000 & 1003.84 & 0.00000 & 0.00000 & -1.25358 \\
\hline 0.00101 & 1004.11 & -0.00336 & -0.00211 & -1.24577 \\
\hline 0.00201 & 1004.35 & -0.00512 & -0.00264 & -1.23643 \\
\hline 0.00265 & 1004.52 & -0.00724 & -0.00399 & -1.23146 \\
\hline 0.00384 & 1004.81 & -0.00963 & -0.00496 & -1.22063 \\
\hline 0.00455 & 1004.98 & -0.01093 & -0.00544 & -1.21407 \\
\hline 0.00985 & 1006.22 & -0.01938 & -0.00799 & -1.16383 \\
\hline 0.01562 & 1007.55 & -0.02856 & -0.01141 & -1.10977 \\
\hline 0.02716 & 1009.87 & -0.02979 & -0.00304 & -0.98791 \\
\hline 0.04010 & 1012.59 & -0.04312 & -0.00852 & -0.87133 \\
\hline 0.05974 & 1016.56 & -0.06355 & -0.02206 & -0.71652 \\
\hline 0.08048 & 1020.52 & -0.08283 & -0.03902 & -0.58333 \\
\hline 0.10837 & 1025.50 & -0.10535 & -0.06299 & -0.45390 \\
\hline 0.15016 & 1032.41 & -0.13950 & -0.10115 & -0.35659 \\
\hline 0.20594 & 1040.55 & -0.17299 & -0.13310 & -0.32679 \\
\hline 0.25133 & 1046.45 & -0.19496 & -0.14878 & -0.33251 \\
\hline 0.29949 & 1052.00 & -0.20552 & -0.15530 & -0.32297 \\
\hline 0.34944 & 1057.32 & -0.22196 & -0.17850 & -0.30288 \\
\hline 0.40170 & 1062.40 & -0.23898 & -0.21846 & -0.26955 \\
\hline 0.44950 & 1066.56 & -0.24538 & -0.25825 & -0.22962 \\
\hline 0.51032 & 1071.20 & -0.23488 & -0.30148 & -0.17097 \\
\hline 0.55198 & 1074.12 & -0.22670 & -0.33644 & -0.13763 \\
\hline 0.60061 & 1077.30 & -0.21687 & -0.38890 & -0.10247 \\
\hline 0.64969 & 1080.25 & -0.20315 & -0.46335 & -0.06286 \\
\hline 0.70096 & 1083.10 & -0.18675 & -0.58251 & -0.01791 \\
\hline 0.74545 & 1085.44 & -0.17504 & -0.73097 & 0.01479 \\
\hline 0.80117 & 1088.05 & -0.14581 & -0.93997 & 0.05129 \\
\hline 0.84704 & 1089.90 & -0.10329 & -1.07598 & 0.07237 \\
\hline 0.89937 & 1091.95 & -0.06239 & -1.11542 & 0.05544 \\
\hline 0.94877 & 1093.83 & -0.03102 & -0.91257 & 0.01658 \\
\hline 1.00000 & 1095.68 & $\begin{array}{l}0.00000 \\
\mathrm{~T}=298.15 \mathrm{~K}\end{array}$ & -0.34466 & 0.00000 \\
\hline 0.00000 & 999.50 & 0.00000 & 0.00000 & -1.36646 \\
\hline 0.00101 & 999.80 & -0.00526 & -0.00389 & -1.36159 \\
\hline 0.00201 & 1000.03 & -0.00654 & -0.00383 & -1.35293 \\
\hline 0.00265 & 1000.19 & -0.00814 & -0.00458 & -1.34823 \\
\hline 0.00384 & 1000.50 & -0.01185 & -0.00673 & -1.34028 \\
\hline 0.00455 & 1000.68 & -0.01383 & -0.00779 & -1.33540 \\
\hline 0.00985 & 1001.95 & -0.02464 & -0.01199 & -1.29633 \\
\hline 0.01562 & 1003.20 & -0.02966 & -0.01029 & -1.24996 \\
\hline 0.02716 & 1005.50 & -0.03074 & 0.00069 & -1.15655 \\
\hline 0.04010 & 1008.20 & -0.04412 & -0.00101 & -1.07596 \\
\hline 0.05974 & 1012.14 & -0.06458 & -0.00674 & -0.97496 \\
\hline 0.08048 & 1016.10 & -0.08582 & -0.01535 & -0.89088 \\
\hline 0.10837 & 1021.00 & -0.10565 & -0.02151 & -0.79796 \\
\hline 0.15016 & 1027.98 & -0.14826 & -0.04829 & -0.71407 \\
\hline 0.20594 & 1036.00 & -0.17807 & -0.06397 & -0.61800 \\
\hline 0.25133 & 1041.84 & -0.19916 & -0.08100 & -0.55115 \\
\hline 0.29949 & 1047.37 & -0.21169 & -0.09937 & -0.47439 \\
\hline 0.34943 & 1052.68 & -0.23094 & -0.13797 & -0.40405 \\
\hline 0.40170 & 1057.68 & -0.24492 & -0.18669 & -0.33165 \\
\hline 0.44950 & 1061.86 & -0.25615 & -0.24015 & -0.27575 \\
\hline 0.51032 & 1066.51 & -0.25038 & -0.29616 & -0.20646 \\
\hline 0.55198 & 1069.38 & -0.24010 & -0.32979 & -0.16729 \\
\hline 0.60061 & 1072.52 & -0.22924 & -0.37076 & -0.13513 \\
\hline 0.64969 & 1075.46 & -0.21726 & -0.41545 & -0.11039 \\
\hline 0.70096 & 1078.29 & -0.20157 & -0.47412 & -0.08529 \\
\hline 0.74545 & 1080.56 & -0.18473 & -0.54612 & -0.06133 \\
\hline 0.80116 & 1083.15 & -0.15588 & -0.67212 & -0.02776 \\
\hline 0.84704 & 1084.99 & -0.11413 & -0.78570 & 0.00715 \\
\hline 0.89937 & 1086.99 & -0.06955 & -0.88745 & 0.02197 \\
\hline 0.94877 & 1088.81 & -0.03290 & -0.80527 & 0.00881 \\
\hline 1.00000 & 1090.63 & $\begin{array}{l}0.00000 \\
\mathrm{~T}=303.15 \mathrm{~K}\end{array}$ & -0.20043 & 0.00000 \\
\hline 0.00000 & 995.15 & 0.00000 & 0.00000 & -1.40895 \\
\hline 0.00101 & 995.46 & -0.00600 & -0.00459 & -1.40501 \\
\hline 0.00201 & 995.70 & -0.00798 & -0.00518 & -1.39720 \\
\hline
\end{tabular}




\begin{tabular}{|c|c|c|c|c|}
\hline$x_{2}$ & $\rho$ & $V_{m}^{E}$ & $\mathbf{V}_{1}{ }^{\mathrm{E}}$ & $\mathbf{V}_{2}{ }^{\mathrm{E}}$ \\
\hline 0.00265 & 995.88 & -0.01085 & -0.00718 & -1.39384 \\
\hline 0.00384 & 996.15 & -0.01231 & -0.00703 & -1.38374 \\
\hline 0.00455 & 996.34 & -0.01497 & -0.00874 & -1.37957 \\
\hline 0.00985 & 997.60 & -0.02578 & -0.01270 & -1.34017 \\
\hline 0.01562 & 998.83 & -0.03016 & -0.01015 & -1.29187 \\
\hline 0.02716 & 1001.11 & -0.03109 & 0.00136 & -1.19366 \\
\hline 0.04010 & 1003.80 & -0.04514 & -0.00078 & -1.10679 \\
\hline 0.05974 & 1007.74 & -0.06754 & -0.00849 & -0.99690 \\
\hline 0.08048 & 1011.65 & -0.08757 & -0.01620 & -0.90294 \\
\hline 0.10837 & 1016.53 & -0.10863 & -0.02378 & -0.80675 \\
\hline 0.15016 & 1023.43 & -0.14956 & -0.04723 & -0.72874 \\
\hline 0.20594 & 1031.50 & -0.18760 & -0.06440 & -0.66265 \\
\hline 0.25133 & 1037.20 & -0.20193 & -0.06764 & -0.60193 \\
\hline 0.29949 & 1042.79 & -0.22270 & -0.08926 & -0.53483 \\
\hline 0.34943 & 1048.08 & -0.24419 & -0.13052 & -0.45583 \\
\hline 0.40170 & 1053.11 & -0.26465 & -0.19250 & -0.37212 \\
\hline 0.44950 & 1057.19 & -0.27067 & -0.24989 & -0.29611 \\
\hline 0.51032 & 1061.82 & -0.26710 & -0.31774 & -0.21850 \\
\hline 0.55198 & 1064.68 & -0.25851 & -0.35553 & -0.17976 \\
\hline 0.60061 & 1067.77 & -0.24578 & -0.39280 & -0.14801 \\
\hline 0.64969 & 1070.66 & -0.23162 & -0.43087 & -0.12418 \\
\hline 0.70096 & 1073.48 & -0.21771 & -0.48871 & -0.10210 \\
\hline 0.74545 & 1075.74 & -0.20219 & -0.56470 & -0.07841 \\
\hline 0.80116 & 1078.28 & -0.17063 & -0.69827 & -0.03968 \\
\hline 0.84704 & 1080.10 & -0.12863 & -0.82328 & -0.00319 \\
\hline 0.89937 & 1082.07 & -0.08279 & -0.94293 & 0.01346 \\
\hline 0.94877 & 1083.84 & -0.04214 & -0.91135 & 0.00479 \\
\hline \multirow{3}{*}{1.00000} & 1085.57 & 0.00000 & -0.50597 & 0.00000 \\
\hline & & $\mathrm{DFM}+\mathrm{NEF}$ & & \\
\hline & & $\mathrm{T}=293.15 \mathrm{~K}$ & & \\
\hline 0.00000 & 951.87 & 0.00000 & 0.00000 & -1.65625 \\
\hline 0.00111 & 952.20 & -0.00396 & -0.00213 & -1.64364 \\
\hline 0.00204 & 952.48 & -0.00755 & -0.00422 & -1.63349 \\
\hline 0.00308 & 952.75 & -0.00828 & -0.00331 & -1.61916 \\
\hline 0.00410 & 953.03 & -0.01011 & -0.00353 & -1.60626 \\
\hline 0.00450 & 953.14 & -0.01087 & -0.00368 & -1.60132 \\
\hline 0.01361 & 955.58 & -0.02432 & -0.00408 & -1.49180 \\
\hline 0.01594 & 956.20 & -0.02774 & -0.00445 & -1.46576 \\
\hline 0.02020 & 957.35 & -0.03594 & -0.00735 & -1.42224 \\
\hline 0.04015 & 962.49 & -0.06189 & -0.01269 & -1.23795 \\
\hline 0.06177 & 967.75 & -0.07807 & -0.01236 & -1.07613 \\
\hline 0.07842 & 971.70 & -0.09127 & -0.01563 & -0.98032 \\
\hline 0.10212 & 977.26 & -0.11796 & -0.03076 & -0.88466 \\
\hline 0.14911 & 987.60 & -0.15733 & -0.05135 & -0.76212 \\
\hline 0.20091 & 998.21 & -0.19588 & -0.07012 & -0.69606 \\
\hline 0.25106 & 1007.71 & -0.22317 & -0.07783 & -0.65676 \\
\hline 0.30142 & 1016.65 & -0.24966 & -0.08713 & -0.62634 \\
\hline 0.35325 & 1025.23 & -0.27129 & -0.10044 & -0.58410 \\
\hline 0.40012 & 1032.61 & -0.29713 & -0.13500 & -0.54019 \\
\hline 0.44808 & 1039.63 & -0.31096 & -0.18246 & -0.46924 \\
\hline 0.50137 & 1047.00 & -0.32677 & -0.27375 & -0.37950 \\
\hline 0.51906 & 1049.29 & -0.32574 & -0.30757 & -0.34257 \\
\hline 0.59582 & 1058.70 & -0.31762 & -0.50318 & -0.19174 \\
\hline 0.65102 & 1064.87 & -0.29669 & -0.66179 & -0.10097 \\
\hline 0.69331 & 1069.14 & -0.25593 & -0.75436 & -0.03545 \\
\hline 0.75499 & 1075.21 & -0.21461 & -0.85747 & -0.00599 \\
\hline 0.79986 & 1079.30 & -0.17306 & -0.85888 & -0.00146 \\
\hline 0.83529 & 1082.48 & -0.14869 & -0.83021 & -0.01431 \\
\hline 0.89267 & 1087.41 & -0.10925 & -0.76760 & -0.03010 \\
\hline 0.93921 & 1091.08 & -0.06015 & -0.82625 & -0.01057 \\
\hline 1.00000 & 1095.68 & $\begin{array}{l}0.00000 \\
\mathrm{~T}=298.15 \mathrm{~K}\end{array}$ & -1.56197 & 0.00000 \\
\hline 0.00000 & 947.78 & 0.00000 & 0.00000 & -1.94746 \\
\hline 0.00111 & 948.11 & -0.00414 & -0.00200 & -1.92954 \\
\hline 0.00204 & 948.40 & -0.00869 & -0.00479 & -1.91585 \\
\hline 0.00308 & 948.67 & -0.00957 & -0.00375 & -1.89679 \\
\hline 0.00410 & 948.94 & -0.01073 & -0.00304 & -1.87854 \\
\hline 0.00450 & 949.05 & -0.01155 & -0.00314 & -1.87185 \\
\hline 0.01361 & 951.48 & -0.02549 & -0.00203 & -1.72641 \\
\hline 0.01594 & 952.09 & -0.02843 & -0.00148 & -1.69190 \\
\hline 0.02020 & 953.23 & -0.03642 & -0.00346 & -1.63488 \\
\hline 0.04015 & 958.34 & -0.06268 & -0.00644 & -1.40702 \\
\hline 0.06177 & 963.75 & -0.09428 & -0.01908 & -1.23642 \\
\hline
\end{tabular}




\begin{tabular}{|c|c|c|c|c|}
\hline $\mathbf{x}_{2}$ & $\rho$ & $V_{m}^{E}$ & $\mathrm{~V}_{1}{ }^{\mathrm{E}}$ & $\mathrm{V}_{2}{ }^{\mathrm{E}}$ \\
\hline 0.07708 & 967.47 & -0.11556 & -0.02942 & -1.14691 \\
\hline 0.10212 & 973.21 & -0.13554 & -0.03454 & -1.02349 \\
\hline 0.14911 & 983.59 & -0.18471 & -0.06465 & -0.86984 \\
\hline 0.20091 & 994.03 & -0.21502 & -0.08815 & -0.71962 \\
\hline 0.25106 & 1003.50 & -0.24578 & -0.12306 & -0.61187 \\
\hline 0.30142 & 1012.32 & -0.26716 & -0.14855 & -0.54204 \\
\hline 0.35325 & 1020.81 & -0.28610 & -0.16178 & -0.51371 \\
\hline 0.40012 & 1028.06 & -0.30442 & -0.16846 & -0.50825 \\
\hline 0.44808 & 1035.03 & -0.31815 & -0.18020 & -0.48807 \\
\hline 0.50097 & 1042.27 & -0.33134 & -0.23281 & -0.42950 \\
\hline 0.51906 & 1044.66 & -0.33679 & -0.26716 & -0.40130 \\
\hline 0.59582 & 1054.00 & -0.32836 & -0.47886 & -0.22627 \\
\hline 0.65102 & 1060.18 & -0.31328 & -0.68267 & -0.11527 \\
\hline 0.69331 & 1064.47 & -0.27817 & -0.79986 & -0.04739 \\
\hline 0.75499 & 1070.47 & -0.23410 & -0.88328 & -0.02343 \\
\hline 0.79986 & 1074.47 & -0.18569 & -0.85511 & -0.01819 \\
\hline 0.83529 & 1077.66 & -0.16518 & -0.84772 & -0.03060 \\
\hline 0.89267 & 1082.46 & -0.11456 & -0.94106 & -0.01519 \\
\hline 0.93921 & 1086.10 & -0.06490 & -1.13614 & 0.00443 \\
\hline 1.00000 & 1090.63 & $\begin{array}{l}0.00000 \\
T=303.15 \mathrm{~K}\end{array}$ & -0.71023 & 0.00000 \\
\hline 0.00000 & 943.68 & 0.00000 & 0.00000 & -2.17412 \\
\hline 0.00111 & 944.01 & -0.00432 & -0.00195 & -2.13922 \\
\hline 0.00204 & 944.30 & -0.00904 & -0.00474 & -2.11187 \\
\hline 0.00308 & 944.58 & -0.01089 & -0.00450 & -2.07905 \\
\hline 0.00410 & 944.84 & -0.01137 & -0.00299 & -2.04618 \\
\hline 0.00450 & 944.95 & -0.01225 & -0.00311 & -2.03427 \\
\hline 0.01361 & 947.36 & -0.02588 & -0.00153 & -1.79066 \\
\hline 0.01594 & 947.97 & -0.02915 & -0.00147 & -1.73760 \\
\hline 0.02020 & 949.11 & -0.03777 & -0.00448 & -1.65233 \\
\hline 0.04015 & 954.18 & -0.06353 & -0.00924 & -1.36119 \\
\hline 0.06177 & 959.56 & -0.09565 & -0.02289 & -1.20096 \\
\hline 0.07708 & 963.25 & -0.11651 & -0.03131 & -1.13658 \\
\hline 0.10212 & 968.95 & -0.13634 & -0.03178 & -1.05571 \\
\hline 0.14911 & 979.28 & -0.18727 & -0.05638 & -0.93421 \\
\hline 0.20083 & 989.66 & -0.21986 & -0.08495 & -0.75673 \\
\hline 0.25106 & 999.11 & -0.25345 & -0.13140 & -0.61754 \\
\hline 0.30142 & 1007.86 & -0.27404 & -0.15961 & -0.53926 \\
\hline 0.35325 & 1016.30 & -0.29390 & -0.16736 & -0.52557 \\
\hline 0.40012 & 1023.50 & -0.31235 & -0.16489 & -0.53343 \\
\hline 0.44808 & 1030.43 & -0.32704 & -0.17365 & -0.51597 \\
\hline 0.50137 & 1037.66 & -0.33916 & -0.23458 & -0.44316 \\
\hline 0.51906 & 1040.00 & -0.34662 & -0.27616 & -0.41191 \\
\hline 0.59582 & 1049.27 & -0.33791 & -0.51495 & -0.21780 \\
\hline 0.65102 & 1055.40 & -0.32223 & -0.72364 & -0.10705 \\
\hline 0.69331 & 1059.61 & -0.28161 & -0.82685 & -0.04042 \\
\hline 0.75499 & 1065.54 & -0.23433 & -0.88640 & -0.02272 \\
\hline 0.79986 & 1069.54 & -0.18908 & -0.85315 & -0.02292 \\
\hline 0.83529 & 1072.71 & -0.16886 & -0.84649 & -0.03525 \\
\hline 0.89267 & 1077.49 & -0.11988 & -0.94311 & -0.02091 \\
\hline 0.93921 & 1081.08 & -0.06723 & -1.13626 & 0.00196 \\
\hline 1.00000 & 1085.56 & $\begin{array}{l}0.00000 \\
\text { (DFM + DMF) }\end{array}$ & -0.92458 & 0.00000 \\
\hline & & $\mathrm{T}=293.15 \mathrm{~K}$ & & \\
\hline 0.00000 & 949.04 & 0.00000 & 0.00000 & -2.32012 \\
\hline 0.00107 & 949.35 & -0.00291 & -0.00040 & -2.34974 \\
\hline 0.00203 & 949.65 & -0.00706 & -0.00222 & -2.37676 \\
\hline 0.00305 & 949.92 & -0.00777 & -0.00045 & -2.40039 \\
\hline 0.00407 & 950.20 & -0.00946 & 0.00040 & -2.42364 \\
\hline 0.00504 & 950.52 & -0.01517 & -0.00283 & -2.44905 \\
\hline 0.01178 & 952.36 & -0.02568 & 0.00462 & -2.56810 \\
\hline 0.01574 & 953.50 & -0.03712 & 0.00426 & -2.62374 \\
\hline 0.02021 & 954.76 & -0.04866 & 0.00543 & -2.67015 \\
\hline 0.04020 & 960.39 & -0.10787 & 0.00251 & -2.74344 \\
\hline 0.06107 & 966.05 & -0.16529 & -0.00220 & -2.67307 \\
\hline 0.08010 & 971.00 & -0.21149 & -0.00749 & -2.55422 \\
\hline 0.10229 & 976.63 & -0.26716 & -0.02322 & -2.40808 \\
\hline 0.14985 & 987.92 & -0.36667 & -0.05841 & -2.11554 \\
\hline 0.19979 & 999.04 & -0.47111 & -0.12600 & -1.85332 \\
\hline 0.25060 & 1009.32 & -0.54761 & -0.20714 & -1.56577 \\
\hline 0.30247 & 1018.90 & -0.60135 & -0.30870 & -1.27623 \\
\hline 0.35085 & 1027.25 & -0.64614 & -0.42024 & -1.06412 \\
\hline 0.40052 & 1035.09 & -0.66808 & -0.51465 & -0.89773 \\
\hline
\end{tabular}




\begin{tabular}{|c|c|c|c|c|}
\hline $\mathbf{x}_{2}$ & $\rho$ & $V_{m}^{E}$ & $\mathrm{~V}_{1}{ }^{\mathrm{E}}$ & $\mathrm{V}_{2}{ }^{\mathrm{E}}$ \\
\hline 0.44895 & 1042.24 & -0.68241 & -0.60267 & -0.78029 \\
\hline 0.50148 & 1049.51 & -0.69247 & -0.71869 & -0.66641 \\
\hline 0.54987 & 1055.56 & -0.67241 & -0.84628 & -0.53007 \\
\hline 0.59758 & 1061.25 & -0.65546 & -1.02630 & -0.40573 \\
\hline 0.65041 & 1067.05 & -0.61797 & -1.22956 & -0.28924 \\
\hline 0.70114 & 1072.06 & -0.55241 & -1.35795 & -0.20905 \\
\hline 0.75060 & 1076.91 & -0.51172 & -1.45524 & -0.19822 \\
\hline 0.80186 & 1081.31 & -0.42572 & -1.56939 & -0.14313 \\
\hline 0.85015 & 1085.35 & -0.35531 & -1.95969 & -0.07252 \\
\hline 0.89870 & 1088.90 & -0.24544 & -2.67869 & 0.02883 \\
\hline 0.95012 & 1092.15 & -0.08810 & -2.97312 & 0.06335 \\
\hline 1.00000 & 1095.68 & $\begin{array}{l}0.00000 \\
\mathrm{~T}=298.15 \mathrm{~K}\end{array}$ & 0.63665 & 0.00000 \\
\hline 0.00000 & 944.30 & 0.00000 & 0.00000 & -2.51916 \\
\hline 0.00107 & 944.62 & -0.00373 & -0.00103 & -2.53234 \\
\hline 0.00203 & 944.91 & -0.00724 & -0.00206 & -2.54396 \\
\hline 0.00305 & 945.18 & -0.00801 & -0.00022 & -2.55287 \\
\hline 0.00407 & 945.47 & -0.01059 & -0.00017 & -2.56312 \\
\hline 0.00504 & 945.78 & -0.01558 & -0.00261 & -2.57512 \\
\hline 0.01178 & 947.61 & -0.02573 & 0.00523 & -2.62372 \\
\hline 0.01574 & 948.75 & -0.03749 & 0.00431 & -2.65069 \\
\hline 0.02021 & 950.01 & -0.04937 & 0.00477 & -2.67375 \\
\hline 0.04020 & 955.62 & -0.10852 & 0.00083 & -2.71936 \\
\hline 0.06107 & 961.28 & -0.16753 & -0.00383 & -2.68451 \\
\hline 0.08010 & 966.23 & -0.21509 & -0.00715 & $\begin{array}{r}-2.60299 \\
\end{array}$ \\
\hline 0.10229 & 971.84 & -0.27063 & -0.01896 & -2.47933 \\
\hline 0.14985 & 983.11 & -0.37146 & -0.05555 & -2.16374 \\
\hline 0.19979 & 994.20 & -0.47630 & -0.13235 & -1.85389 \\
\hline 0.25060 & 1004.48 & -0.55549 & -0.21713 & -1.56732 \\
\hline 0.30247 & 1014.05 & -0.61071 & -0.30704 & -1.31101 \\
\hline 0.35085 & 1022.40 & -0.65762 & -0.40937 & -1.11695 \\
\hline 0.40052 & 1030.22 & -0.67950 & -0.51083 & -0.93196 \\
\hline 0.44895 & 1037.33 & -0.69154 & -0.62416 & -0.77425 \\
\hline 0.50148 & 1044.59 & -0.70222 & -0.77268 & -0.63218 \\
\hline 0.54987 & 1050.64 & -0.68332 & -0.90460 & -0.50219 \\
\hline 0.59758 & 1056.22 & -0.65589 & -1.04146 & -0.39625 \\
\hline 0.65041 & 1062.04 & -0.62137 & -1.19562 & -0.31272 \\
\hline 0.70114 & 1067.10 & -0.56184 & -1.31899 & -0.23911 \\
\hline 0.75060 & 1071.87 & -0.51295 & -1.46600 & -0.19628 \\
\hline 0.80186 & 1076.34 & -0.43522 & -1.65994 & -0.13259 \\
\hline 0.85015 & 1080.37 & -0.36407 & -1.97172 & -0.08071 \\
\hline 0.89870 & 1083.85 & -0.24588 & -2.36918 & -0.00654 \\
\hline 0.95012 & 1087.40 & -0.12467 & -2.67276 & 0.00910 \\
\hline 1.00000 & 1090.63 & 0.00000 & -1.87364 & 0.00000 \\
\hline 0.00000 & 939.53 & 0.00000 & 0.00000 & -2.83829 \\
\hline 0.00107 & 939.85 & -0.00383 & -0.00080 & -2.82663 \\
\hline 0.00203 & 940.15 & -0.00824 & -0.00251 & -2.81762 \\
\hline 0.00305 & 940.42 & -0.00908 & -0.00052 & -2.80485 \\
\hline 0.00407 & 940.70 & -0.01090 & 0.00046 & -2.79369 \\
\hline 0.00504 & 941.01 & -0.01600 & -0.00195 & -2.78676 \\
\hline 0.01178 & 942.87 & -0.02908 & 0.00309 & -2.72860 \\
\hline 0.01574 & 944.00 & -0.04033 & 0.00231 & -2.70590 \\
\hline 0.02021 & 945.24 & -0.05089 & 0.00343 & -2.68382 \\
\hline 0.04020 & 950.84 & -0.11078 & -0.00483 & -2.64043 \\
\hline 0.06107 & 956.49 & -0.17051 & -0.01185 & -2.61014 \\
\hline 0.08010 & 961.43 & -0.21857 & -0.01510 & -2.55518 \\
\hline 0.10229 & 967.03 & -0.27479 & -0.02665 & -2.45249 \\
\hline 0.14985 & 978.29 & -0.37775 & -0.07227 & -2.11090 \\
\hline 0.19979 & 989.35 & -0.48292 & -0.16960 & -1.73784 \\
\hline 0.25060 & 999.61 & -0.56292 & -0.26905 & -1.44171 \\
\hline 0.30247 & 1009.11 & -0.61388 & -0.34617 & -1.23124 \\
\hline 0.35085 & 1017.50 & -0.66655 & -0.43304 & -1.09859 \\
\hline 0.40052 & 1025.31 & -0.68918 & -0.52041 & -0.94179 \\
\hline 0.44895 & 1032.40 & -0.70075 & -0.63765 & -0.77821 \\
\hline 0.50148 & 1039.64 & -0.71093 & -0.80149 & -0.62091 \\
\hline 0.54987 & 1045.69 & -0.69310 & -0.93252 & -0.49711 \\
\hline 0.59758 & 1051.26 & -0.66554 & -1.03022 & -0.41996 \\
\hline 0.65041 & 1057.05 & -0.62869 & -1.10314 & -0.37367 \\
\hline 0.70114 & 1062.12 & -0.57083 & -1.16562 & -0.31731 \\
\hline 0.75060 & 1066.86 & -0.51915 & -1.31806 & -0.25370 \\
\hline 0.80186 & 1071.55 & -0.46727 & -1.61895 & -0.18270 \\
\hline 0.85015 & 1075.36 & -0.37088 & -1.94587 & -0.09327 \\
\hline
\end{tabular}




\begin{tabular}{|c|c|c|c|c|}
\hline $\mathbf{x}_{2}$ & $\rho$ & $V_{m}^{E}$ & $\mathbf{V}_{1}{ }^{\mathrm{E}}$ & $\mathbf{V}_{2}{ }^{\mathrm{E}}$ \\
\hline 0.89870 & 1078.87 & -0.25615 & -2.21982 & -0.03480 \\
\hline 0.95012 & 1082.52 & -0.14719 & -2.47449 & -0.02502 \\
\hline 1.00000 & 1085.57 & $\begin{array}{l}0.00000 \\
(\mathrm{DFM}+\mathrm{DMA})\end{array}$ & -3.47870 & 0.00000 \\
\hline & & $\mathrm{T}=293.15 \mathrm{~K}$ & & \\
\hline 0.00000 & 941.53 & 0.00000 & 0.00000 & -4.10693 \\
\hline 0.00102 & 941.85 & -0.00886 & -0.00474 & -4.05106 \\
\hline 0.00203 & 942.12 & -0.01304 & -0.00494 & -3.99316 \\
\hline 0.00319 & 942.44 & -0.01891 & -0.00640 & -3.93037 \\
\hline 0.00416 & 942.73 & -0.02608 & -0.00998 & -3.88215 \\
\hline 0.00512 & 942.97 & -0.02836 & -0.00877 & -3.83075 \\
\hline 0.01014 & 944.20 & -0.03911 & -0.00276 & -3.58909 \\
\hline 0.01488 & 945.43 & -0.05627 & -0.00573 & -3.40313 \\
\hline 0.02106 & 946.91 & -0.06725 & 0.00001 & -3.19390 \\
\hline 0.03965 & 951.64 & -0.13262 & -0.02165 & -2.82072 \\
\hline 0.06127 & 956.87 & -0.19069 & -0.03222 & -2.61877 \\
\hline 0.08031 & 961.24 & -0.22603 & -0.02556 & -2.52165 \\
\hline 0.10829 & 967.90 & -0.31471 & -0.05494 & -2.45366 \\
\hline 0.15051 & 977.27 & -0.40857 & -0.07858 & -2.27096 \\
\hline 0.20771 & 989.17 & -0.50644 & -0.13769 & -1.91300 \\
\hline 0.24943 & 997.55 & -0.58228 & -0.22458 & -1.65868 \\
\hline 0.30098 & 1007.36 & -0.65859 & -0.35003 & -1.37520 \\
\hline 0.35107 & 1016.08 & -0.68648 & -0.45273 & -1.11854 \\
\hline 0.40899 & 1025.70 & -0.71431 & -0.58436 & -0.90208 \\
\hline 0.44904 & 1032.00 & -0.72245 & -0.66650 & -0.79111 \\
\hline 0.50099 & 1039.89 & -0.73297 & -0.77455 & -0.69156 \\
\hline 0.55685 & 1047.80 & -0.71792 & -0.88726 & -0.58317 \\
\hline 0.60252 & 1053.93 & -0.69491 & -1.01447 & -0.48409 \\
\hline 0.65092 & 1060.14 & -0.66303 & -1.20409 & -0.37288 \\
\hline 0.70818 & 1067.07 & -0.60921 & -1.46879 & -0.25500 \\
\hline 0.75093 & 1071.89 & -0.54922 & -1.63689 & -0.18845 \\
\hline 0.80734 & 1077.93 & -0.45813 & -1.79467 & -0.13918 \\
\hline 0.85560 & 1082.91 & -0.38002 & -1.98442 & -0.10925 \\
\hline 0.90937 & 1087.99 & -0.25970 & -2.50644 & -0.03580 \\
\hline 0.94691 & 1091.29 & -0.15910 & -3.04155 & 0.00252 \\
\hline 1.00000 & 1095.68 & $\begin{array}{l}0.00000 \\
\mathrm{~T}=298.15 \mathrm{~K}\end{array}$ & -2.25263 & 0.00000 \\
\hline 0.00000 & 936.94 & 0.00000 & 0.00000 & -4.20745 \\
\hline 0.00102 & 937.27 & -0.01002 & -0.00579 & -4.14934 \\
\hline 0.00203 & 937.54 & -0.01431 & -0.00602 & -4.08834 \\
\hline 0.00319 & 937.85 & -0.01933 & -0.00652 & -4.02118 \\
\hline 0.00416 & 938.15 & -0.02763 & -0.01117 & -3.97131 \\
\hline 0.00512 & 938.41 & -0.03198 & -0.01196 & -3.91931 \\
\hline 0.01014 & 939.60 & -0.03924 & -0.00214 & -3.66209 \\
\hline 0.01488 & 940.84 & -0.05790 & -0.00639 & -3.46863 \\
\hline 0.02106 & 942.31 & -0.06843 & 0.00001 & -3.25006 \\
\hline 0.03965 & 947.01 & -0.13276 & -0.02009 & -2.86204 \\
\hline 0.06127 & 952.23 & -0.19188 & -0.03102 & -2.65663 \\
\hline 0.08031 & 956.68 & -0.23690 & -0.03338 & -2.56745 \\
\hline 0.10829 & 963.22 & -0.31621 & -0.05272 & -2.48581 \\
\hline 0.15051 & 972.60 & -0.41470 & -0.08106 & -2.29770 \\
\hline 0.20771 & 984.47 & -0.51396 & -0.14219 & -1.93206 \\
\hline 0.24943 & 992.83 & -0.59094 & -0.22942 & -1.67879 \\
\hline 0.30098 & 1002.61 & -0.66777 & -0.35283 & -1.39922 \\
\hline 0.35107 & 1011.30 & -0.69551 & -0.45310 & -1.14357 \\
\hline 0.40899 & 1020.91 & -0.72558 & -0.58878 & -0.92325 \\
\hline 0.44904 & 1027.21 & -0.73585 & -0.67984 & -0.80458 \\
\hline 0.50099 & 1034.92 & -0.74139 & -0.80124 & -0.68178 \\
\hline 0.55685 & 1042.89 & -0.72885 & -0.94674 & -0.55544 \\
\hline 0.60252 & 1048.79 & -0.69422 & -1.08615 & -0.43567 \\
\hline 0.65092 & 1054.90 & -0.66181 & -1.28092 & -0.32980 \\
\hline 0.70818 & 1061.62 & -0.59826 & -1.48933 & -0.23109 \\
\hline 0.75093 & 1066.56 & -0.54281 & -1.58996 & -0.19548 \\
\hline 0.80734 & 1072.56 & -0.45653 & -1.67996 & -0.16457 \\
\hline 0.85560 & 1077.33 & -0.38070 & -1.95571 & -0.11490 \\
\hline 0.90937 & 1082.62 & -0.25763 & -2.78471 & -0.00579 \\
\hline 0.94691 & 1086.09 & -0.14024 & -3.35752 & 0.04015 \\
\hline 1.00000 & 1090.63 & $\begin{array}{l}0.00000 \\
\mathrm{~T}=303.15 \mathrm{~K}\end{array}$ & -0.50403 & 0.00000 \\
\hline 0.00000 & 932.33 & 0.00000 & 0.00000 & -4.21568 \\
\hline 0.00102 & 932.68 & -0.01220 & -0.00795 & -4.16990 \\
\hline 0.00203 & 932.95 & -0.01661 & -0.00825 & -4.11837 \\
\hline 0.00319 & 933.25 & -0.02076 & -0.00783 & -4.06024 \\
\hline
\end{tabular}




\begin{tabular}{lllll}
\hline $\mathbf{X}_{\mathbf{2}}$ & $\boldsymbol{\rho}$ & $\boldsymbol{V}_{\boldsymbol{m}}^{\mathbf{E}}$ & $\mathbf{V}_{\mathbf{1}}^{\mathbf{E}}$ & $\mathbf{V}_{\mathbf{2}}^{\mathbf{E}}$ \\
\hline 0.00416 & 933.56 & -0.03021 & -0.01356 & -4.01913 \\
0.00512 & 933.80 & -0.03267 & -0.01238 & -3.97229 \\
0.01014 & 934.98 & -0.03935 & -0.00143 & -3.74289 \\
0.01488 & 936.22 & -0.05854 & -0.00556 & -3.56616 \\
0.02106 & 937.69 & -0.06961 & 0.00111 & -3.35731 \\
0.03965 & 942.36 & -0.13285 & -0.01662 & -2.94849 \\
0.06127 & 947.60 & -0.19606 & -0.03272 & -2.69886 \\
0.08031 & 952.10 & -0.24788 & -0.04368 & -2.58630 \\
0.10829 & 958.54 & -0.31965 & -0.05648 & -2.48665 \\
0.15051 & 967.90 & -0.41974 & -0.08237 & -2.32380 \\
0.20771 & 979.75 & -0.52139 & -0.14135 & -1.97098 \\
0.24943 & 988.09 & -0.59946 & -0.23359 & -1.70041 \\
0.30098 & -0.67783 & -0.36440 & -1.40577 \\
0.35107 & -0.70642 & -0.46108 & -1.15990 \\
0.40899 & -0.73656 & -0.58261 & -0.95902 \\
0.44904 & 1006.52 & -0.74677 & -0.67066 & -0.84014 \\
0.50099 & 1016.10 & -0.75379 & -0.80927 & -0.69854 \\
0.55685 & 1022.38 & -0.74041 & -0.98076 & -0.54913 \\
0.60252 & 1030.19 & -0.71096 & -1.12506 & -0.43778 \\
0.65092 & 1038.07 & -0.67471 & -1.27546 & -0.35255 \\
0.70818 & 1044.11 & -0.60956 & -1.41996 & -0.27563 \\
0.75093 & 1050.25 & -0.55303 & -1.52995 & -0.22899 \\
0.80734 & 1057.05 & -0.49455 & -1.78531 & -0.18653 \\
0.85560 & 1061.88 & -0.38518 & -2.12074 & -0.09227 \\
0.90937 & 1068.17 & -0.26543 & -2.70102 & -0.02270 \\
0.94691 & 1072.87 & -0.16076 & -3.04404 & 0.00090 \\
1.00000 & 1077.94 & 0.00000 & -2.63786 & 0.00000 \\
\hline
\end{tabular}

Figures 2, 3, 4 and 5 show that for each of the (DFM + NMF or NEF or DMF or DMA) binary systems, an increase in temperature $(293.15$ to 303.15$) \mathrm{K}$, resulted in an increase in the negative magnitude of the $V_{m}^{E} v s x_{2}$ isotherms over the entire $x_{2}$ - range. This effect may be resulting from a more favorable mutual geometrical fitting of component molecules into the expanded cavities of the unlike component liquid structure in binary mixture. Another plausible explanation for the observed increase in the negative magnitude of $V_{m}^{E}$ values is as follows. Increase in temperature leads to increase in kinetic energy which would promote dissociation of self- associated species of pure components (amide-amide or DFM-DFM) as well as the DFM-amide complex associates and would positively contribute to $V_{m}^{E}$ values. The observed contraction in $V_{m}{ }^{E}$ values may suggest that an increase in temperature causes a greater dissociation effect on the amideamide or DFM-DFM self-associates than on the formation of DFM-amide complexes. As result, the equilibrium would be shifted more favorably towards the DFM-amide complex formation leading to closer molecular packing and more negative $V_{m}{ }^{E}$ values.

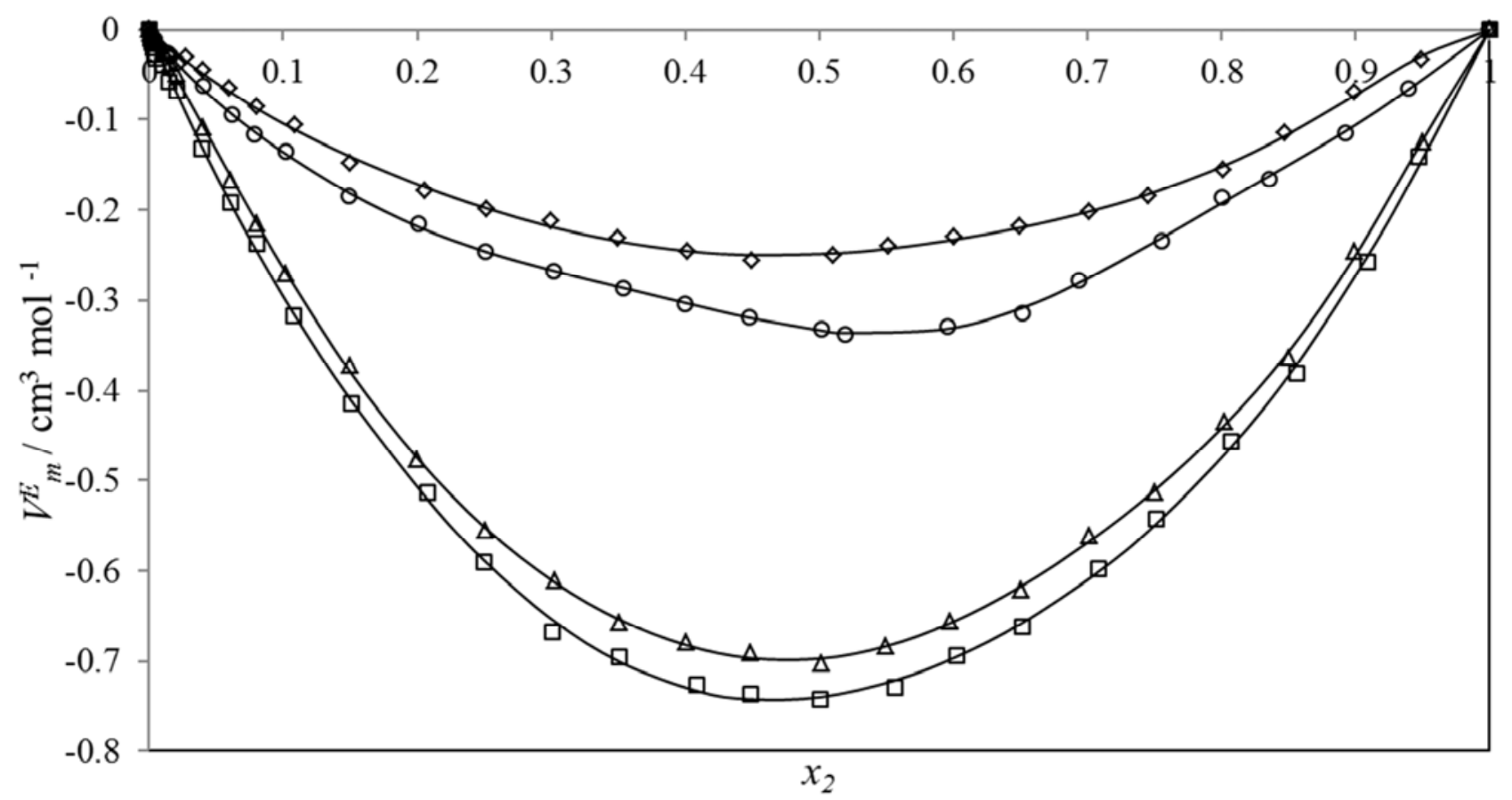

Figure 1. Excess molar volume, $V_{m}^{E}$ vs $x_{2}$ for the [DFM (2) + amides (1)] binary mixtures: ( () NMF; ( () NEF; ( $\left.\Delta\right)$ DMF and () DMA at 298.15 K. Each solid line has been calculated from the Redlich-Kister fitting correlation coefficients (Table 3). 

Mixtures at $293.15 \mathrm{~K}, 298.15 \mathrm{~K}$ and $303.15 \mathrm{~K}$

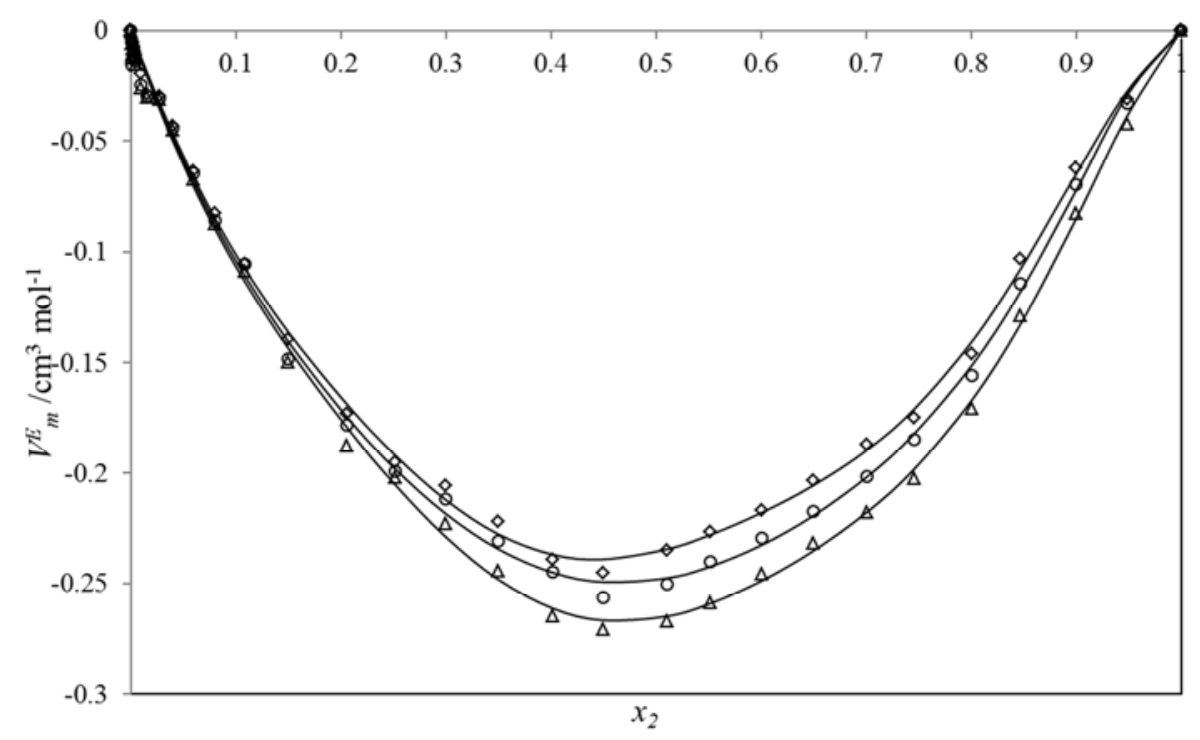

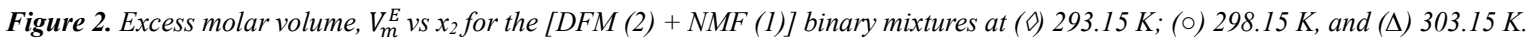

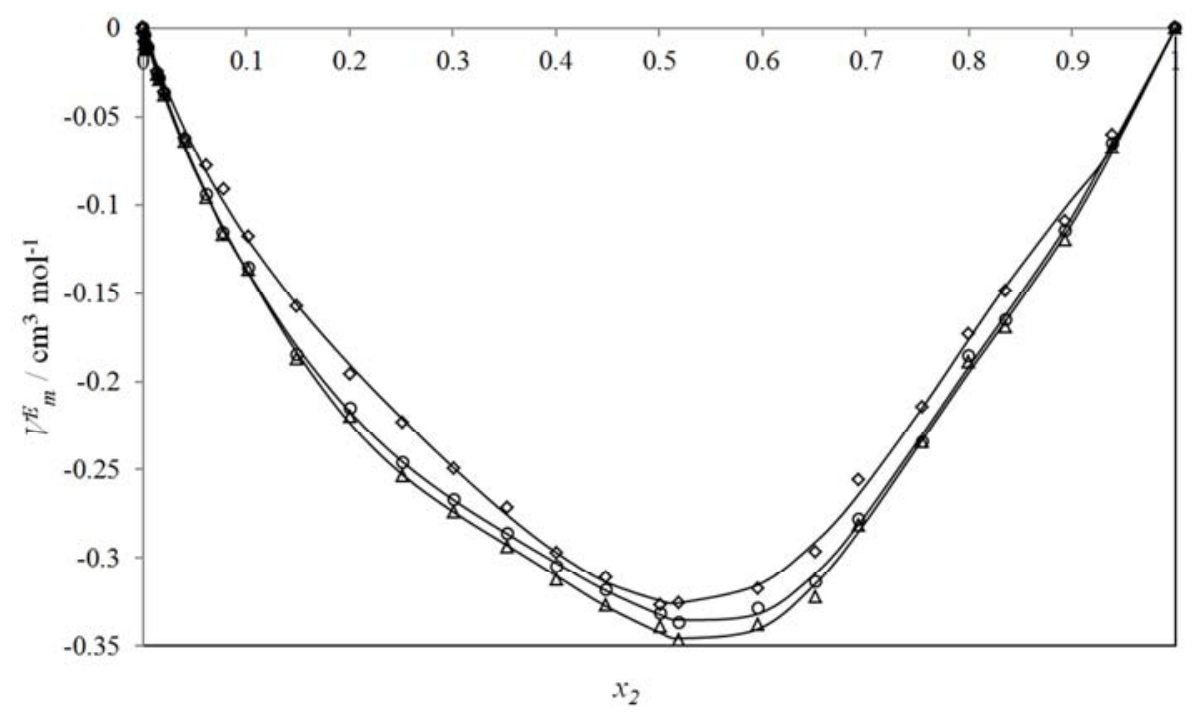

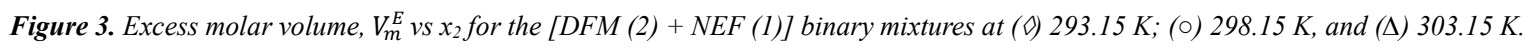

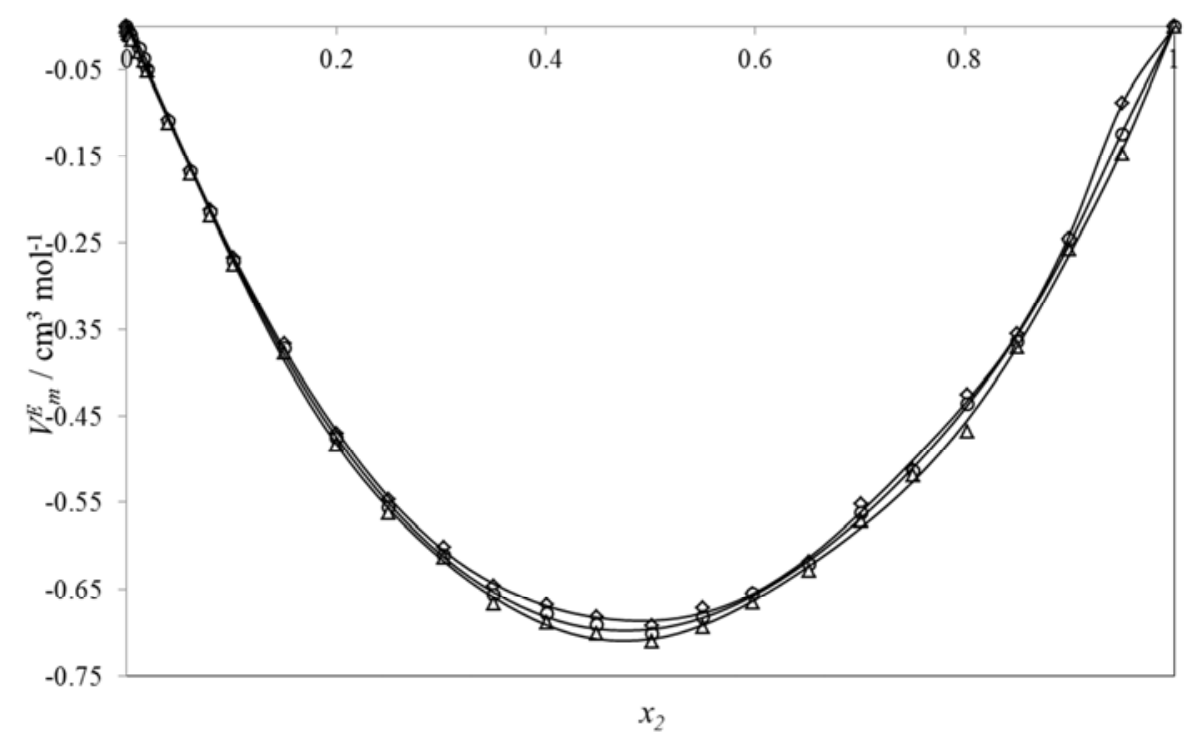

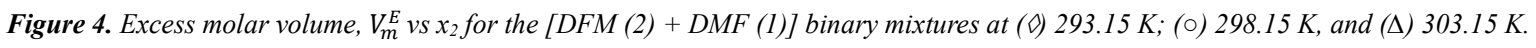




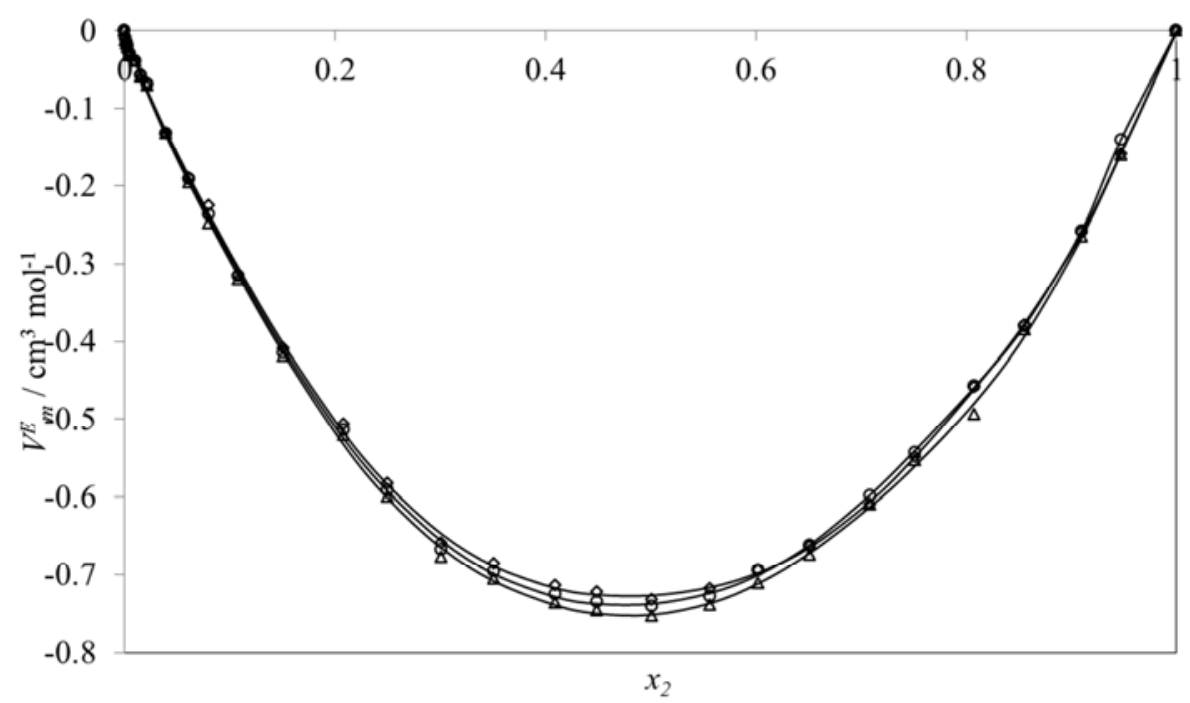

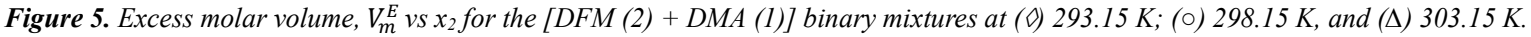

\subsection{Excess Partial Molar Volumes $\left(\bar{V}_{i}^{E}\right)$}

Differential properties such as excess partial molar volumes $\left(V_{i}^{E}\right)$ separate the two component contributions in the binary system and are more sensitive to changes in the aggregation schemes arising from the mixing process. The $V_{i}^{E}$ values of component $i$ in a mixture describe the rate of change of the excess molar volume with composition, and provide information on the individual component response to the intermolecular interactions. For each binary system the $V_{1}^{E}$ and $V_{2}^{E}$ values have been obtained in accordance with

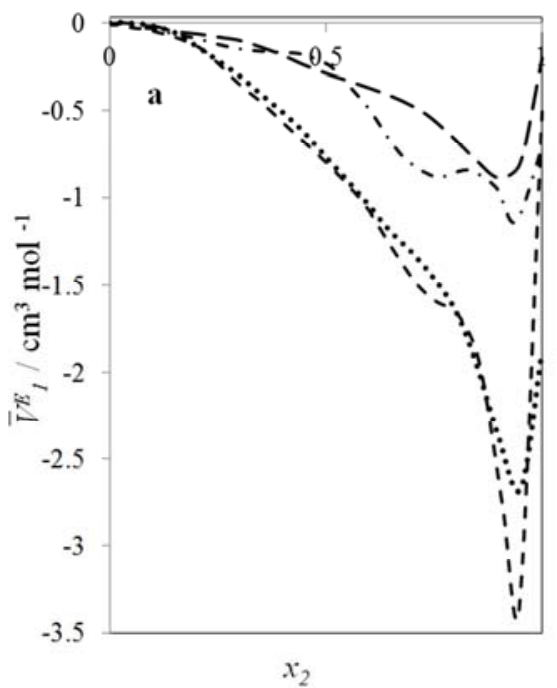

equations (4) and (5) respectively [23]:

$$
\begin{gathered}
\bar{V}_{1}^{E}=V_{m}^{E}-\mathrm{x}_{2}\left(\partial V_{m}^{E} / \partial \mathrm{x}_{2}\right)_{\mathrm{P}, \mathrm{T}} \\
\bar{V}_{2}^{E}=V_{m}^{E}+\left(1-\mathrm{x}_{2}\right)\left(\partial V_{m}^{E} / \partial \mathrm{x}_{2}\right)_{\mathrm{P}, \mathrm{T}}
\end{gathered}
$$

The optimized Redlich-Kister polynomial least squares fitting correlation coefficients (Table 3 ) for a specific binary system at a given temperature have been used to evaluate the $\left(\partial V_{m}^{E} / \partial x_{2}\right)_{P, T}$ function over the entire composition range.

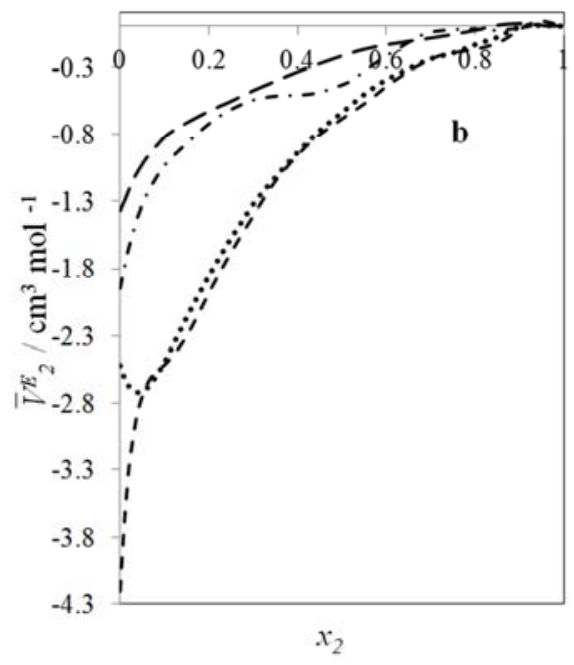

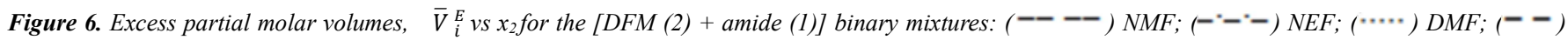
DMA at $298.15 \mathrm{~K}$.

Table 2 shows the calculated excess partial molar volumes, $V_{i}^{E}$, for components in each of the four binary systems at $(\mathrm{T}=$ 293.15, 298.15 and 303.15) K. Figures 6 (a) and (b) represent the excess partial molar volumes for amides, $\left(V_{1}^{E}\right)$ and that for DFM, $\left(V_{2}^{E}\right)$ respectively in (DFM + NMF or NEF or DMF or DMA) binary systems at $298.15 \mathrm{~K}$. Negative $V_{i}^{E}$ values were observed for both components of each of the four binary systems over the entire $x_{2}$-range. Negative
$V_{i}^{E}$ values signify that both components are more closely packed in the binary mixture than in their pure states. The negative magnitude of the $\bar{V}_{i}^{E}$ function in the infinite dilute regions of the four binary systems is in the order: DMA > DMF $>$ NEF $>$ NMF. Negative $\bar{V}_{i}^{E}$ values corroborate the $V_{m}^{E}$ data (Figure 1) and suggest enhancement of the DFMamide, dipole-dipole interactions and possible mutual structural effects arising from the geometrical interstitial 
accommodation of each component into the bulk liquid structure of second component. Therefore for each binary system, the solute-solvent interactions are stronger than the intermolecular interactions in the pure components. Table 3 shows that the negative magnitude of $\bar{V}_{i}^{E}$ values for a component in each of (DFM + NMF or NEF or DMF or DMA) binary mixtures increases with the rise in temperature as was observed for the $V_{m}^{E}$ data (Figures 2-5).

\subsection{Limiting Excess $\left(\bar{V}_{i}^{E, \infty}\right)$ and Partial $\left(\bar{V}_{i}^{\infty}\right)$ Molar Volumes}

Information about the solute-solvent intermolecular interactions and their dependence on temperature can be obtained from the limiting excess partial molar volumes $\left(\bar{V}_{i}^{E, \infty}\right)$ of each component in a mixture [24]. The optimized Redlich-Kister polynomial fitting correlation coefficients for each of (DFM + NMF or NEF or DMF or DMA) binary systems at $(\mathrm{T}=293.15,298.15$ and 303.15) $\mathrm{K}$ (Table 3) have been used to obtain values of the limiting excess partial molar volumes for an amide $\left(\bar{V}_{1}^{E, \infty}\right)$ and DFM $\left(\bar{V}_{2}^{E, \infty}\right)$ using Equations (6) and (7) [24] respectively,

$$
\begin{aligned}
\bar{V}_{1}^{E, \infty}= & \sum_{k=\text { even }} A_{k}-\sum_{k=\text { odd }} A_{k} \\
& \bar{V}_{2}^{E, \infty}=\sum_{k} A_{k}
\end{aligned}
$$

Table 4 lists the calculated $\bar{V}_{i}^{E, \infty}$ data for components in each binary system. A negative value for the $\bar{V}_{i}^{E, \infty}$ function was obtained for each component in each binary mixture at the three temperatures, further suggesting the enhancement molecular parking efficiency and supporting the observed negative $V_{m}^{E}$ data. Thus in the infinite dilute solutions of DFM or amide in each of the four binary systems, the solutesolvent interactions are stronger than the intermolecular interactions in the pure components which gives rise to greater packing efficiency.

The limiting partial molar volume, $\bar{V}_{i}^{\infty}$ of component $i$ in a mixture can be calculated from the expression: $\bar{V}_{i}^{\infty}=\bar{V}_{i}^{E, \infty}+V_{m, i}^{*}$, where $V_{m, i}^{*}$ is the molar volume of the pure component. It can be observed that values of both $V_{1}^{\infty}$ and $V_{2}^{\infty}$ (Table 4) show an increase with the rise in temperature.

Table 3. Least square fitted $\left(A_{k}\right)$ coefficients of Equation (4) and corresponding standard deviation of fit $\sigma\left(V_{m}^{E}\right)$ for the $(D F M+N M F$ or $N E F$ or DMF or DMA) binary systems at $(T=293.15,298.15$ and 303.15)K.

\begin{tabular}{lllll}
\hline & NMF & \multicolumn{1}{l}{ NEF } & DMF & DMA \\
\hline Coefficients & & \multicolumn{2}{l}{$T=293.15$} & $K$ \\
$A_{0}$ & -0.943 & -1.297 & -2.746 & -2.909 \\
$A_{1}$ & -0.227 & 0.222 & -0.090 & -0.158 \\
$A_{2}$ & -0.119 & 0.623 & -0.339 & -1.022 \\
$A_{3}$ & 0.892 & -1.139 & -2.031 & 0.017 \\
$A_{4}$ & 0.178 & -0.350 & 2.007 & 3.525 \\
$A_{5}$ & -2.045 & 0.870 & 7.892 & -2.082 \\
$A_{6}$ & 0.085 & -0.585 & -7.257 & -6.179 \\
$A_{7}$ & 0.926 & & -8.181 & 8.202 \\
$A_{8}$ & & & 7.493 & 3.405 \\
$A_{9}$ & & & 0.932 & -6.906 \\
$\sigma\left(V_{m}^{E}\right)$ & 0.005 & 0.004 & 0.005 & 0.006 \\
$A_{0}$ & -0.994 & -1.328 & -2.786 & -2.953 \\
\hline
\end{tabular}

\begin{tabular}{lllll}
\hline & NMF & NEF & DMF & DMA \\
\hline$A_{1}$ & -0.140 & 0.399 & -0.265 & -0.230 \\
$A_{2}$ & -0.021 & 0.201 & -0.037 & -0.644 \\
$A_{3}$ & 0.383 & -2.914 & -0.093 & 0.428 \\
$A_{4}$ & -2.310 & 0.533 & -0.674 & 3.019 \\
$A_{5}$ & -0.735 & 5.177 & 1.485 & -0.352 \\
$A_{6}$ & 0.462 & -2.611 & 0.106 & -8.051 \\
$A_{7}$ & -0.091 & -3.281 & -1.450 & 7.813 \\
$A_{8}$ & & 1.876 & 1.195 & 6.273 \\
$A_{9}$ & & & & -8.655 \\
$\sigma\left(V_{m}^{E}\right)$ & 0.006 & 0.003 & 0.004 & 0.006 \\
& & $T=303.15$ & $K$ & \\
$A_{0}$ & -1.063 & -1.369 & -2.831 & -3.009 \\
$A_{1}$ & -0.154 & 0.427 & -0.344 & -0.210 \\
$A_{2}$ & 0.030 & 0.462 & 0.470 & -0.210 \\
$A_{3}$ & 0.777 & -3.365 & 1.103 & 0.867 \\
$A_{4}$ & -0.282 & -0.889 & -4.844 & -1.069 \\
$A_{5}$ & -1.426 & 6.351 & -1.926 & 4.460 \\
$A_{6}$ & 0.358 & 0.247 & 9.451 & 1.856 \\
$A_{7}$ & 0.352 & -4.038 & 1.487 & -4.172 \\
$A_{8}$ & & & -5.404 & -0.994 \\
$\sigma\left(V_{m}^{E}\right)$ & 0.006 & 0.003 & 0.005 & 0.008 \\
\hline
\end{tabular}

Table 4. Limiting excess $\left(\bar{V}_{i}^{E, \infty}\right)$ and partial $\left(\bar{V}_{i}^{\infty}\right)$ molar volumes for the components in [DFM + (NMF or NEF or DMF or DMA)] binary systems at $(T=293.15,298.15$ and 303.15) K derived from Equations (8) and (9).

\begin{tabular}{llll}
\hline $\mathbf{T} / \mathbf{K}$ & $\mathbf{2 9 3 . 1 5 K}$ & $\mathbf{2 9 8 . 1 5}$ & $\mathbf{3 0 3 . 1 5}$ \\
\hline $\bar{V}_{1}^{E, \infty}$ & $(\mathrm{DFM}+\mathrm{NMF})$ & & -0.506 \\
$\bar{V}_{2}^{E, \infty}$ & -0.345 & -0.200 & -1.409 \\
$\bar{V}_{1}^{\infty}$ & -1.254 & -1.366 & 58.850 \\
$\bar{V}_{2}^{\infty}$ & -1.409 & 58.897 & 135.071 \\
$\bar{V}_{1}^{E, \infty}$ & 133.966 & 134.483 & \\
$\bar{V}_{2}^{E, \infty}$ & $(\mathrm{DFM}+\mathrm{NEF})$ & & -0.925 \\
$\bar{V}_{1}^{\infty}$ & -1.562 & -0.710 & -2.174 \\
$\bar{V}_{2}^{\infty}$ & -1.656 & -1.947 & 76.531 \\
$\bar{V}_{1}^{E, \infty}$ & 75.228 & 76.411 & 134.306 \\
$\bar{V}_{2}^{E, \infty}$ & 133.564 & 133.902 & \\
$\bar{V}_{1}^{\infty}$ & $(\mathrm{DFM}+\mathrm{DMF})$ & & -3.479 \\
$\bar{V}_{2}^{\infty}$ & -0.637 & -1.874 & -2.838 \\
$\bar{V}_{1}^{E, \infty}$ & -2.320 & -2.519 & 74.319 \\
$\bar{V}_{2}^{E, \infty}$ & 77.656 & 75.531 & 133.642 \\
$\bar{V}_{1}^{\infty}$ & 133.172 & 133.331 & \\
$\bar{V}_{2}^{\infty}$ & $(\mathrm{DFM}+\mathrm{DMA})$ & & -2.638 \\
\hline & -2.253 & -0.504 & -4.216 \\
\hline & -4.107 & -4.207 & 90.806 \\
\hline
\end{tabular}

The $\bar{V}_{i}^{\infty}$ value for a component in a liquid mixture has two contributing terms: one due to the intrinsic molar volume, $V_{\text {int }}$, of the non-solvated solute molecule and the other due to the co-sphere, $V_{s}$, which arises from the interaction of the solute with solvent molecules. For a polyatomic solute molecule the change in $V_{\text {int }}$ with temperature is negligible. Therefore any observed temperature dependence of $\bar{V}_{i}^{\infty}$ indicates how the co-sphere $V_{s}$ depends on temperature [25]. In this study, the observed increase in $\bar{V}_{1}^{\infty}$ and $\bar{V}_{2}^{\infty}$ is attributed to the positive contribution from $V_{s}$ which increases with temperature due to a decrease in the interaction between the unlike molecules as a result of the increase in Brownian motion. Since for each of the $[\mathrm{DFM}+(\mathrm{NMF}$ or $N \mathrm{EF}$ or DMF or DMA $)]$ binary systems, the negative magnitude of $V \underset{m}{E}$ values slightly 
increases with temperature rise, it is possible that the geometric effects more than offset the entropic expansion effects reflected in the increase of $\bar{V}_{i}^{\infty}$ values.

\section{Conclusions}

In this study, measured density values of pure DFM, NMF, NEF, DMF and DMA as well as their (DFM + NMF or NEF or DMF or DMA) binary mixtures over the entire composition range are reported at $(\mathrm{T}=293.15,298.15$ and 303.15) K. These data have been used to derive: the excess molar volumes of solution, $V_{m}^{E}$, excess partial molar volumes $\bar{V}_{i}^{E}$, limiting excess partial molar volumes $\bar{V}_{i}^{E, \infty}$, and limiting partial molar volumes $\left(V_{i}{ }^{\infty}\right)$ of each component of a binary mixture. The excess molar volumes for each of (DFM + NMF or NEF or DMF or DMA) binary systems were negative over the entire composition range at the three temperatures investigated. The negative excess molar volumes observed for each of the four binary systems have been discussed in terms of specific interactions and structural effects. The magnitude of the negative deviation in $V_{m}{ }^{E}$ values follows the order: DMA $>$ DMF $>$ NEF $>$ NMF. The results indicated that the increase in temperature had a greater effect on component self-association than on complex formation between molecules of components in (DFM + NMF or NEF or DMF or DMA) binary mixture. Calculated excess partial molar volumes, $\bar{V}_{i}{ }^{E}$, limiting excess partial molar volumes $\bar{V}_{i}^{E, \infty}$ and limiting partial molar volumes $V_{i}{ }^{\infty}$ of each component in (DFM + NMF or NEF or DMF or DMA) binary mixture complimented the $V_{m}^{E}$ data.

\section{Acknowledgement}

Financial support from the University of Botswana is gratefully acknowledged.

\section{References}

[1] Mokate, O., Ddamba, W. A. A., Volumetric properties of difurylmethane in methanol from 288.15 to $308.15 \mathrm{~K}$. Journal of Solution Chemistry, 34 (2005) 1327-1339.

[2] Mokate, O., Ddamba, W. A. A., Volumetric properties of (Difurylmethane + Alkan-1-ol) binary mixtures at 298.15K. Journal of Solution Chemistry, 35 (2006) 1493-1503.

[3] Mokate, O., Ddamba, W. A. A., Effect of temperature on volumetric properties of [Difurylmethane $+\left(C_{2}-C_{6}\right)$ Alkan-1ol] binary systems: Analyses of new literature density data in the temperature range 288.15-308.15K. Journal of Solution Chemistry, 37 (2008) 331-350.

[4] Mmereki, B. T., Oathotse, I., Ddamba, W. A. A., Ultrasonic speeds and isentropic compressibilities of \{Difurylmethane + $\left(C_{I}-C_{6}\right)$ Alkan-1-ol $\}$ binary mixtures at $T=298.15 \mathrm{~K}$. Journal of Chemical Thermodynamics, 42 (2010) 1346-1351.

[5] Zarei, H., Golroudbari, A. S., Behroozi, M, Experimental studies on volumetric and viscometric properties of binary and ternary mixtures of N,N-dimethylacetamide, Nmethylformamide, and propane-1,2-diol at different temperatures. Journal of Molecular Liquids, 187 (2013) 260265.

[6] Nain, K. A., Densities and volumetric properties of (acetonitrile + an amide) binary mixtures at temperatures between $293.15 \mathrm{~K}$ and $318.15 \mathrm{~K}$. Journal of Chemical Thermodynamics, 38 (2006) 1362-1370.

[7] Garcia, B., Alcalde, R., Leal, M. J., Solute-Solvent interactions in amide-water mixed solvents. Journal of Physical Chemistry, 101 (1997) 7991-7997.

[8] Gadzuric, S., Nikolic, A., Vranes, M., Jovic, B., Damjanovic, M., Dozic, S., Volumetric properties of binary mixtures of Nethylformamide with tetrahydrofuran, 2-butanone, and ethylacetate from $T=(293.15$ to 313.15$)$ K. Journal of Chemical Thermodynamics, 51 (2012) 37-44.

[9] Jovic, B., Nikolic, A., Kordic, B., Densitometric and spectroscopic investigation of interactions of selected Nsubstituted amides and acetonitrile. Journal of Molecular Liquids, 191 (2014) 10-15.

[10] Sengwa, R. J., Sankhla, S., Khatri, V., Choudhary, S., Static permittivity and molecular interactions in binary mixtures of ethanolamine with alcohols and amides. Fluid Phase Equilibria, 293 (2010) 137-140.

[11] Nain, A. K., Densities, ultrasonic speeds, viscosities and excess properties of binary mixtures of methyl methacrylate with $\mathrm{N}, \mathrm{N}$-dimethylformamide and $\mathrm{N}, \mathrm{N}$-dimethylacetamide at different temperatures. Journal of Chemical Thermodynamics, 60 (2013) 105-116

[12] Nikolic, A., Jovic, B., Krstic, V., Trickovic, J, Excess molar volumes of N-methylformamide + tetrahydropyran, + 2pentanone + n-propylacetate at the temperatures between 298.15K and 313.15K. Journal of Molecular Liquids, 133 (2007) 39-42.

[13] Scharlin, P., Steinby, K., Domanska, U., Volumetric properties of binary mixtures of $\mathrm{N}, \mathrm{N}$-dimethylformamide with water or water- $\mathrm{d}_{2}$ at temperatures from $277.13 \mathrm{~K}$ to $318.15 \mathrm{~K}$. Journal of Chemical Thermodynamics, 34 (2002) 927-957.

[14] Weissberger, A., Organic Solvents: Physical Properties and Methods of Purification, New York, USA: John Wiley \& Sons (1986) 55-57.

[15] Pandey, K., Awasthi, A., Awasthi, A, Acoustic, volumetric and spectroscopic investigations in binary mixtures of formamide/ $\mathrm{N}$-methylformamide +2 -chloroethanol at various temperatures. Journal of Molecular Liquids, 189 (2013) 343349 .

[16] Sharma, V. K., Dua, R., Sharma, D, Excess molar volumes and excess isentropic compressibilities of binary and ternary mixtures of $o$-chlorotoluene with cyclic ether and amides or cyclohexane at different temperatures. Journal of Chemical Thermodynamics, 78 (2014) 241-253.

[17] Almasi, M., Thermodynamic properties of binary mixtures containing N,N-dimethylformamide +2 -alkanol. Cubic and statistical associating fluid theory-based equation of state analysis. Taiwan Institute of Chemical Engineers, 45 (2014) 365-371.

[18] Pal, A., Sharma, S., Kumar, H, Volumetric properties of binary mixtures of 2-alkoxyethanols with N, N-dimethylformamide and N, N-dimethylacetamide at $298.15 \mathrm{~K}$. Journal of Molecular Liquids, 108 (2003) 231-255. 
[19] Peshwe, A., Arbad, B., Pachaling, S, Volumetric and viscometric studies on $\mathrm{N}, \mathrm{N}$-dimethylacetamide and methanol binary mixtures at different temperatures. Journal of Chemical Science, 7 (2009) 1505-1517.

[20] Buchwalter. S. L. Polymer Chemistry. Journal of Polymer Science: Polymer Chemistry Edition, 23 (1985) 2879-3098.

[21] Redlich, O. and Kister, T. A., Algebraic representation of thermodynamic properties and the classification of solutions. Ind. Eng. Chem. 40, 345-348 (1948).

[22] Ivanov, E., Abrosimov, V. K., Lebedeva, E. Y., Apparent molar volume and expansivities of $\mathrm{H}_{2} \mathrm{O}$ and $\mathrm{D}_{2} \mathrm{O}$ in $\mathrm{N}, \mathrm{N}-$ dimethylformamide and $\mathrm{N}, \mathrm{N}$-dimethyacetamide in the range of $\mathrm{T}=(278.15$ to 318.15$) \mathrm{K}$ at $\mathrm{p}=0.1 \mathrm{MPa}$ : A comparative analysis Journal of Chemical Thermodynamics, 53 (2012) 131-139.

[23] Douhẻret, G., Davis, M. I., Measurement, analysis, and utility

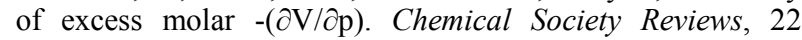
(1993) 43-50.

[24] Acree Jr, W. E., Thermodynamic properties of nonelectrolytes, Orlando, Florida, USA: Academic Press (1984) 15-19.

[25] Reading, J. F., Watson, I. D., Hedwig, G. R., Thermodynamic properties of peptide solutions 5. Partial molar volumes of glycylglycine, glycyl-DL-leucine, and glycyl-DL-serine at 308.15 and 318.15K. Journal of Chemical Thermodynamics, 22 (1990) 159-165. 